\title{
Exploration of CT manifestations of different clinical types of novel coronavirus pneumonia
}

\author{
Xue-Ying Li ${ }^{1 \#}$, Yang Zhou ${ }^{2 \#} \wedge$, Zi-Xuan Kong ${ }^{1} \wedge$, Mei-Dan Hou ${ }^{1}$, Ning Luo $^{1 \wedge}$, Wei-Hang Sun ${ }^{1 \wedge}$, \\ Nan Huang ${ }^{3} \wedge$, Chao Yang ${ }^{1}$, Ao-Dan Zhang ${ }^{1}$, Yu-Shi Li ${ }^{1}$ \\ ${ }^{1}$ Department of Radiology, The Second Affiliated Hospital of Dalian Medical University, Dalian, China; ${ }^{2}$ Department of Ultrasound, The First \\ Affiliated Hospital of Dalian Medical University, Dalian, China; ${ }^{3}$ Medical Imaging Center, Taihe Hospital, Shiyan, China \\ Contributions: (I) Conception and design: XY Li, Y Zhou, MD Hou, ZX Kong, N Luo, C Yang; (II) Administrative support: None; (III) Provision of \\ study materials or patients: None; (IV) Collection and assembly of data: Y Zhou, XY Li, MD Hou, N Luo, N Huang, C Yang, AD Zhang; (V) Data \\ analysis and interpretation: ZX Kong, WH Sun, YS Li; (VI) Manuscript writing: All authors; (VII) Final approval of manuscript: All authors. \\ \#These authors contributed equally to this work. \\ Correspondence to: Zi-Xuan Kong. No. 467, Zhongshan Road, Dalian, China. Email: drzxkong@163.com.
}

Background: To explore computed tomography (CT) characteristics of the 2019 novel coronavirus (COVID-19) pneumonia and explore variations among the different clinical types.

Methods: Clinical and CT imaging data of 43 patients diagnosed with COVID-19 in our hospital and the cooperative hospital between January 15-30, 2020 were collected (27 male and 16 female). Patients were classified as common type (26 cases, 60\%), severe type (14 cases, 33\%) or critical type (three cases, $7 \%$ ) according to the new coronavirus pneumonia treatment scheme (sixth edition). Patient clinical data and CT images were analyzed and evaluated.

Results: Fever was the main symptom in common type COVID-19 cases (23/26, 88.46\%). Both severe and critical type COVID-19 patients had fever and cough symptoms, and dyspnea was observed in all three critical COVID-19 patients. CT manifestations in the common type COVID-19 cohort were bilateral involvement (20/26, 71\%), multiple lesions (14/26, 54\%), ground-glass density shadow (17/26, 65\%), and some cases were accompanied by local consolidation $(9 / 26,35 \%)$, which is consistent with early stage COVID-19 CT performance. CT manifestations in the severe and critical types involved both lungs. Severe COVID-19 cases predominantly consisted of multiple mixed-density lesions (10/14, 71\%), and a few patients showed diffuse lung glass density shadows in both lungs (4/14, 29\%), which is consistent with the progression stage COVID-19 CT performance. Critical COVID-19 cases exhibited mixed-density lesions, and two cases displayed "white lung", which is the CT manifestation at the severe COVID-19 stage. Only one critical COVID-19 patient had pleural effusion.

Conclusions: The CT manifestations of COVID-19 are specific and there are variations between different clinical types. Thus, CT is an important clinical tool for early diagnosis and assessment of the severity of COVID-19.

Keywords: 2019 novel coronavirus (COVID-19); computed tomography (CT); imaging characteristics

Submitted Aug 17, 2020. Accepted for publication Nov 03, 2020.

doi: $10.21037 /$ apm-20-1811

View this article at: http://dx.doi.org/10.21037/apm-20-1811

\footnotetext{
^ ORCID ID: Yang Zhou, 0000-0002-8501-2611; Zi-Xuan Kong, 0000-0002-7494-3322; Ning Luo, 0000-0002-9144-7363; Wei-Hang Sun, 0000-0001-7808-7837; Nan Huang, 0000-0003-2570-4998; Ao-Dan Zhang, 0000-0002-6291-6271.
} 


\section{Introduction}

Since the outbreak of the coronavirus disease 2019 (COVID-19), a disease caused by severe acute respiratory syndrome Coronavirus 2 (SARS-CoV-2), SARS-CoV is prone to large-scale spread as a result of the failure of timely detection and early isolation due to its long incubation time and strong infectiousness (1), it has spread rapidly throughout the world. According to the sixth edition of COVID-19 diagnosis and treatment program issued by the National Health Commission, diagnostic criteria needs to be combined with epidemiology, clinical and computed tomography (CT) manifestations, and COVID-19 nucleic acid detection (2). COVID-19 nucleic acid detection is considered to be the gold standard for the diagnosis of the disease. At present, routine test specimens are collected from pharynx swabs, which result in a high false negative rate. The diagnostic process is further protracted due to the need for multiple examinations.

Chest CT scans provide several advantages including convenience, timeliness, and direct display of focus. CT examination is valuable for early diagnosis of suspected COVID-19 cases as well as observation of the progress and outcome of the disease. This paper aims to summarize and discuss CT manifestations of COVID-19 in different clinical types, and to provide important reference value for early clinical diagnosis, isolation, and treatment.

We present the following article in accordance with the MDAR reporting checklist (available at http://dx.doi. org/10.21037/apm-20-1811).

\section{Methods}

This investigation was a retrospective study and was approved by our institutional review board. The informed consent requirement was waived. All procedures performed in this study involving human participants were in accordance with the Declaration of Helsinki (as revised in 2013).

\section{General materials}

Clinical and imaging data of COVID-19 patients diagnosed in our hospital and the cooperative hospital between January 15-30, 2020 were retrospectively collected. Inclusion criteria: (I) patients with respiratory tract specimens who tested positive by COVID-19 nucleic acid detection; (II) patients performing chest CT examination for the first time without any previous treatment; (III) patients without other infectious diseases; (IV) patients without artifacts in CT scanning images and meeting the observation conditions. There were 43 COVID-19 patients eligible for inclusion in the study, including 27 males and 16 females, with an onset age of $28-74$ years and median age of 53 years.

The sixth edition of the COVID-19 diagnosis and treatment program divides COVID-19 into mild, common, severe, and critical types (1). Mild type COVID-19 patients were not included in this study due to mild clinical symptoms and no abnormal pneumonia manifestations on imaging examination. Among the 43 COVID-19 patients included in this study, 26 were common type, 14 were severe type, and three were critical type. The patients' clinical symptoms, peripheral blood routine examination results (white blood cell (WBC), lymphocyte ratio), and hypersensitive C-reactive protein results were recorded.

\section{CT examination method}

Thoracic CT examination was performed in 43 patients using the Siemens64 and Philips128 spiral scanners. Patients wore a surgical mask throughout the examination and were in a supine position. The scans were conducted with patients entering the scanner head first and breath-holding. The scan ranged from the chest entrance to the lung floor. The scanning parameters were as follows: tube voltage $120 \mathrm{kV}$, automatic tube current, layer thickness $5 \mathrm{~mm}$, layer spacing $1 \mathrm{~mm}$, and matrix $512 \times 512$.

\section{CT image analysis}

Image analysis and evaluation were conducted by two senior medical practitioners in the radiologic cardiothoracic department. In cases of disagreement, consensus was achieved through consultation. The following CT manifestations were recorded for each patient: (I) scope of involvement: single or double (bilateral) lung; (II) distribution of lesions: peripheral, central, or simultaneous involvement; (III) number of lesions: single, double, or multiple; (IV) lesion opacity: ground-glass, solid, or mixed opacity; (V) other thoracic manifestations: Mediastinum and hilar lymphadenopathy, pleural thickening, and pleural effusion. 


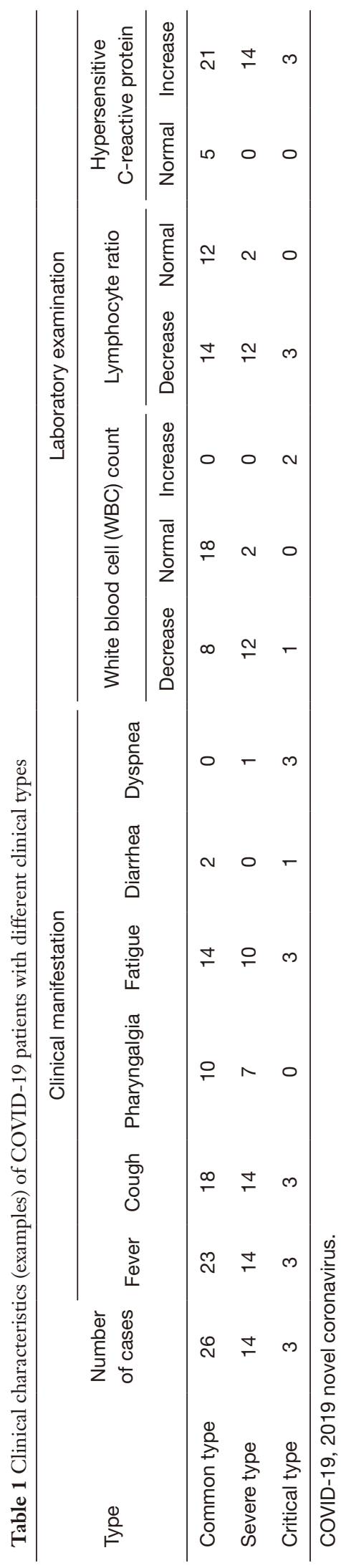

\section{Statistical analysis}

Statistical analysis data were analyzed by SPSS 22.0 software. The median age was used. The imaging manifestations and lesions were statistically analyzed.

\section{Results}

\section{Clinical features of COVID-19 patients}

Among the 43 COVID-19 patients included in this study, two common-type patients without obvious clinical discomfort were diagnosed during quarantine due to close contact with confirmed cases. The remaining 41 patients had different clinical symptoms (Table 1). Twenty patients $(20 / 43,46.5 \%)$ had normal peripheral blood leukocyte count, 21 patients $(21 / 43,48.8 \%)$ had decreased leukocyte count, and 29 patients $(29 / 43,67.4 \%)$ had decreased lymphocyte proportion. Also, 38 patients (38/43, 88.4\%) had high levels of hypersensitive C-reactive protein. The clinical characteristics of COVID-19 patients with different clinical types are shown in Table 1.

\section{CT manifestations of COVID-19 patients}

Of the 43 COVID-19 patients included in this study, six $(6 / 43,13.9 \%)$ had common lesions located in one side of the lung field (i.e. single lung involvement), while the remaining 37 patients $(37 / 43,86.1 \%)$ exhibited bilateral involvement. In common type patients, the lesions were mainly distributed in the peripheral zone $(19 / 26,73.1 \%$, Figure 1), with single $(7 / 26,26.9 \%)$, double $(5 / 26,19.2 \%)$ or multiple $(14 / 26,53.9 \%)$ lesions. In 17 common type patients $(17 / 26,65.4 \%)$, the lesions showed ground-glass opacity, in which there was thickening or "paving stone sign" of vascular bundles. The other nine common type patients $(9 / 26,34.6 \%)$ exhibited ground-glass opacity and consolidation, and no solid lesions were found.

The lesions of the severe and critical patients had simultaneous distribution in peripheral and central zones. Three severe type patients $(3 / 14,21.4 \%)$ exhibited groundglass opacity (Figure 2), one severe case $(1 / 14,7.1 \%)$ showed solid opacity focus, while the remaining severe and critical patients displayed mixed opacity focus (Figures 3,4). Mediastinal lymphadenopathy was observed in two severe and critical type patients $(2 / 43,4.7 \%)$, and pleural effusion was found in one case $(1 / 43,2.3 \%)$. The CT manifestations of COVID-19 patients for each clinical classification are shown in Table 2. 

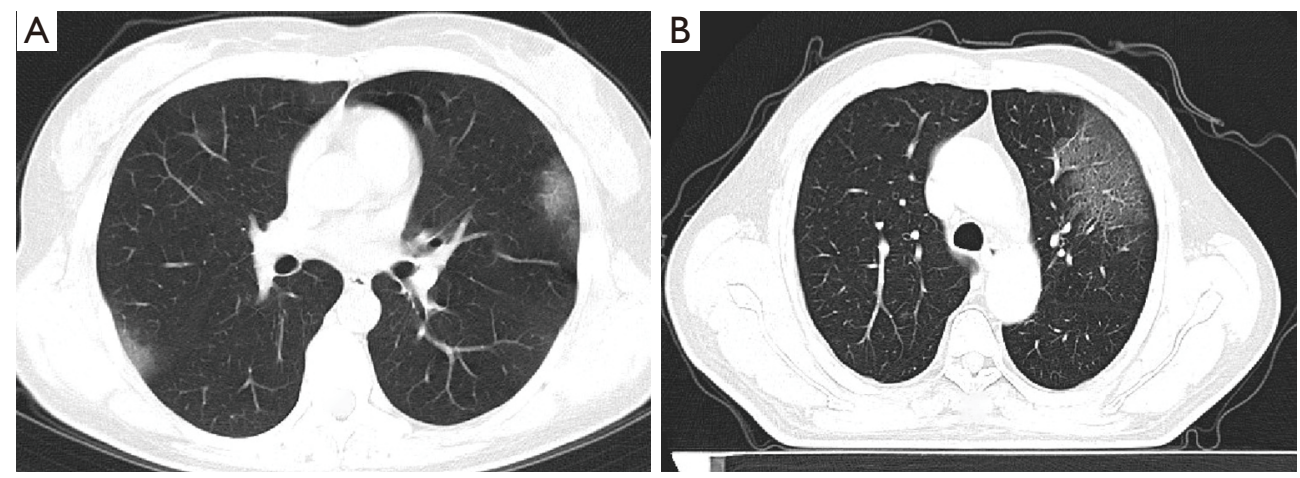

Figure 1 Common type COVID-19 CT performance. (A) Female, 46 years old, with fever for 3 days. CT shows patchy ground-glass density in the subpleural area of the upper lobe of both lungs and thickened small blood vessels. (B) Male, 34 years old, with fever and cough for 6 days. CT shows a large sheet of ground glass density shadow in the upper lobe of the left lung. Also, a grid shadow can be seen inside, showing a paving stone sign. COVID-19, 2019 novel coronavirus; CT, computed tomography.
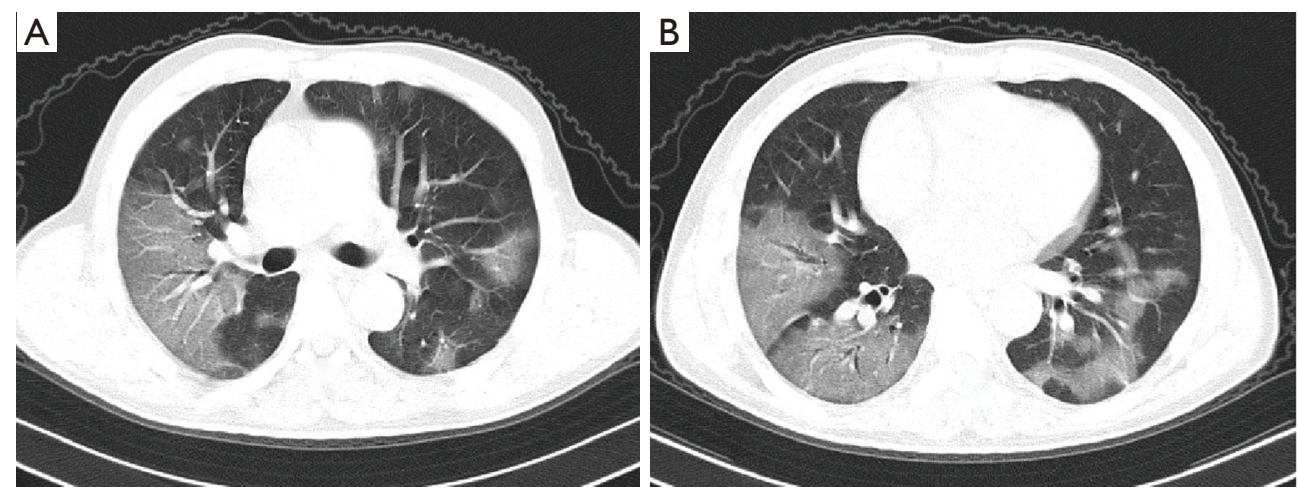

Figure 2 Severe type COVID-19 CT performance. (A,B) Male, 52 years old; fever for 5 days with diarrhea. CT shows multiple pieces of ground-glass density on both lungs, with the right lung as the focus. COVID-19, 2019 novel coronavirus; CT, computed tomography.
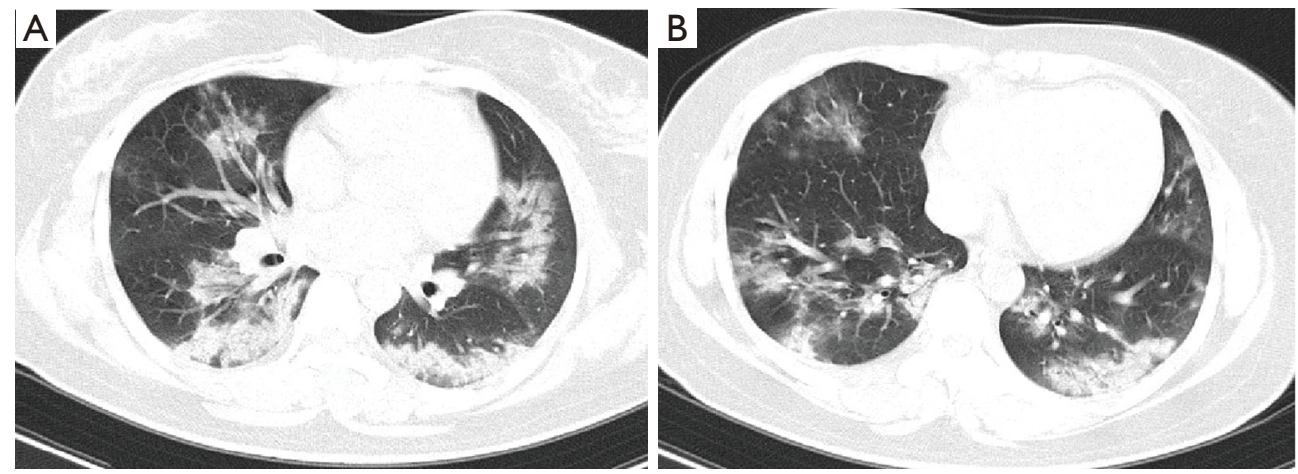

Figure 3 Severe type COVID-19 CT performance. (A,B) Female, 28 years old, with fever and cough for 7 days. CT shows multiple spotted ground-glass density and consolidation shadows in both lungs, with the lower lobe of the lungs as the focus. Bronchial meteorology can be seen in local lesions. COVID-19, 2019 novel coronavirus; CT, computed tomography. 

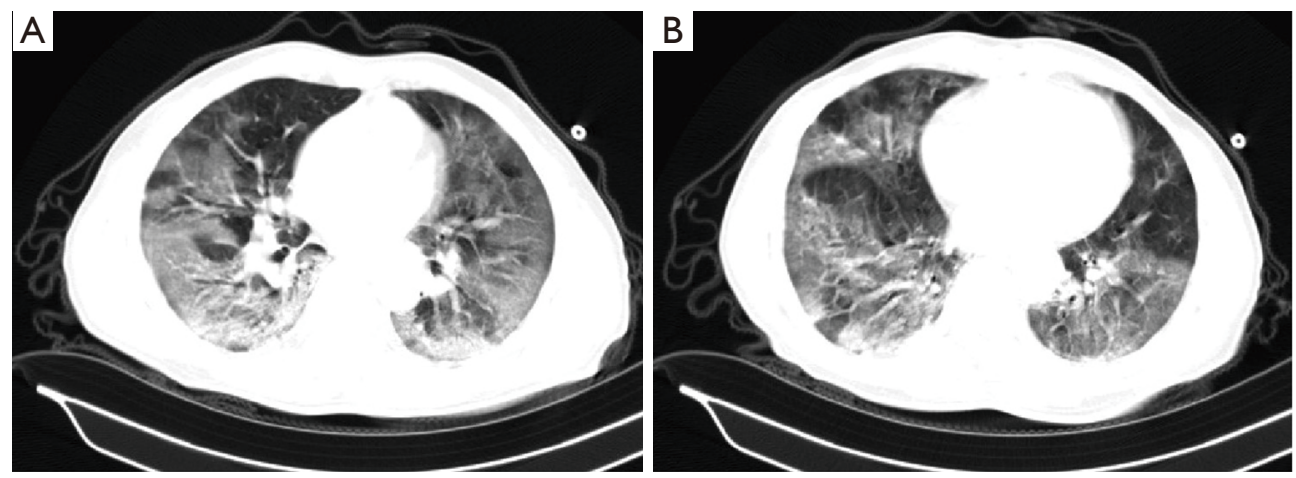

Figure 4 Critical type COVID-19 CT performance. (A,B) Male, 69 years old, fever and dry cough for 6 days. CT shows multiple diffuse ground-glass densities and consolidation in both lungs. White lung can be seen. COVID-19, 2019 novel coronavirus; CT, computed tomography.

\section{Discussion}

2019-nCoV belongs to the $\beta$ genus of coronaviruses, with studies showing that it shares more than $85 \%$ homology with the severe acute respiratory syndrome (SARS)-like coronaviruses carried by bats. Recent epidemiological studies report that the incubation period of COVID-19 is 1-14 days (mostly 3-7 days), with fever, fatigue, and dry cough being the main symptoms. According to our analysis of 43 COVID-19 patients in this study, the primary symptom (present in $93 \%$ of patients) was fever, which is consistent with the recent research (3), suggesting that fever can be an important index of clinical triage. However, the clinical symptoms of COVID-19 varied. In this study, there were also patients that only exhibited atypical symptoms such as asthenia or diarrhea. The clinical symptoms of severe and critical patients were more obvious, and all critical patients experienced dyspnea. This may be due to the fact that a more severe disease leads to an increased amount of exudate in the alveoli as well as a wider range of involvement, resulting in decreased pulmonary ventilation function and ultimately, symptoms of dyspnea (4).

Notably, $4.6 \%$ of the common type patients in this study had no obvious clinical symptoms, and returned a negative COVID-19 nucleic acid test. However, chest CT examination in this cohort showed that there were spot-like ground-glass opacity lesions under the pleura of the lung. These patients were subsequently confirmed as COVID-19 positive after multiple nucleic acid tests, suggesting that such patients were occult and the diagnosis was easily missed. Chest CT screening is therefore essential for patients with a history of epidemiological exposure.

In laboratory tests, Huang et al. demonstrated that $63 \%$ of COVID-19 patients exhibited a reduced lymphocyte ratio, and that $85 \%$ of intensive care unit (ICU) patients had a decreased lymphocyte ratio. This was higher than that of common type COVID-19 patients (54\%) (5), and is consistent with the results of the present study. Furthermore, Huang et al. reported a 25\% WBC count reduction in COVID-19 patients, which is lower than the result observed in the present study (48.8\%). This may be due to the fact that WBC count is easily affected by the age and physiological factors of patients (6). In this study, two patients with severe disease exhibited an increased leukocyte count. However, this is likely owing to the fact that these two patients were elderly men that had low immunity and a history of chronic bronchitis, which may have been caused by a combination of other bacterial infections.

The CT manifestations of COVID-19 are similar to those of SARS and the Middle East respiratory syndrome (MERS), which are caused by other coronaviruses. The lesions are primarily interstitial inflammation of the lung (7-9) that mainly manifest as patchy or nodular groundglass and local consolidation opacities. According to the COVID-19 Imaging Diagnosis Guidelines (2020 first edition), issued by the infectious disease group at the Chinese Medical Association's Chinese Society of Radiology, COVID-19 can be divided into early, progression, severe, and outcome stages based on the onset time and course of the disease. Early stage COVID-19 CT typically shows multiple patchy ground-glass opacity in both lungs, which could be accompanied by local consolidation shadows mostly distributed along the bronchovascular bundle and subpleural zones. Thickening blood tube shadows are also seen. In the progressive stage of the disease, there are 


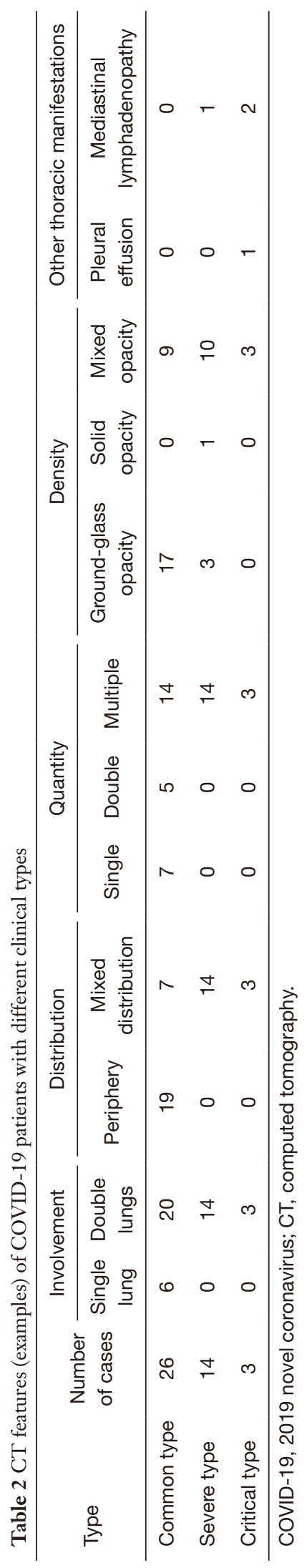

numerous manifestations in the lung, including groundglass opacity, consolidation, nodule, and cord. These are primarily distributed in the central and peripheral areas of the lung, the subpleural zone, and the base of the lung. There are also fibrosis lesions at this stage of the disease, although no significant lymphadenopathy is seen. In the severe stage, lesions can develop into diffuse consolidation in both lungs, and non-consolidated areas can exhibit patchy ground-glass shadow. When the majority of both lungs are involved, they present "white lung", which is occasionally accompanied by a small amount of pleural effusion.

Based on this, 26 cases of common type COVID-19 were mainly characterized by early stage CT manifestations, mostly involving both lungs. Patchy or nodular groundglass density shadow can be seen in the lung, primarily distributed in the subpleural area, with thickening of pulmonary vessels and paving stone signs. The pathological mechanism of COVID-19 is not presently clear. However, considering the SARS coronavirus, which shares $85 \%$ homology with COVID-19, the early virus colonizes alveolar and respiratory bronchioles epithelium (10), resulting in alveolar wall edema. Serous exudate appears in the alveoli, the CT image shows as a ground-glass shadow, and the "paving stone" sign may appear when the alveolar interstitial inflammatory thickens. As their condition deteriorates, severe type COVID-19 patients can experience acute diffuse lung injury (11), with more inflammatory exudate in the alveoli. CT shows a wider range of lesions and coexisting properties, such as ground-glass shadow, consolidation, and cord. All of these are CT manifestations in the progressive stage.

The sixth edition of COVID-19 diagnosis and treatment program pointed out that the patients with obvious lesion progression $(>50 \%)$ in $24-48$ hours were regarded as severe patients (2). In this study, the three severe COVID-19 cases were elderly men. Two of these cases were further complicated with acute respiratory distress syndrome (ARDS). The main CT manifestation at the severe stage is "white lung", suggesting that elderly patients are more likely to experience rapid and serious disease progression. Thus, if the CT examination displays diffuse lung disease or signs of white lung, the risk of ARDS should be altered clinically and early symptomatic support treatment should be administered. After treatment, the clinical symptoms of the patients in this group (42/43, 97.8\%) gradually improved. The CT showed that the absorption of the lesion was reduced, the density became low, and the lung consolidation changed to ground glass opacity with fiber- 
stripe shadow.

COVID-19 is mainly distinguished from pneumonia caused by various types of viral pneumonia and other types of pathogens. (I) SARS, MERS, influenza virus: Because the pathological changes of viral pneumonia are similar, it is difficult to distinguish the imaging findings. The identification mainly depends on clinical and pathogenic examinations. Most SARS infected patients can have obvious clinical symptoms in a short time Symptoms, such as dyspnea, recurrence, or persistent fever, and the disease progresses quickly; MERS can induce kidney damage, so it progresses faster than SARS, and can quickly progress to ARDS, MOF or even death (12). (II) Bacterial pneumonia: Bacterial pneumonia usually has an acute onset, with increased peripheral white blood cell counts, and the lesions occur in the lung parenchyma. Most of them are bronchial pneumonia and lobar pneumonia, which are the consolidation of the distribution of lung lobes or lung segments (13). (III) Mycoplasma pneumonia is manifested as ground glass shadow, lung lobe or lung segment consolidation shadow, visible pleural thickening, pleural effusion and hilar lymph node enlargement are more common (14). In addition, COVID-19 needs to be differentiated from non-infectious diseases such as vasculitis, connective tissue-related lung disease, cryptogenic organizing pneumonia, alveolar proteinosis, and cardiogenic pulmonary edema.

In summary, CT manifestations of COVID-19 are specific, and there are relative differences between each of the COVID-19 clinical classifications. Thus, CT is an important clinical tool for early screening, diagnosis, and severity assessment during the course of the disease. However, due to the limited number of cases analyzed in this study, further research into the clinical features and imaging findings of COVID-19 is needed.

\section{Acknowledgments}

Funding: None.

\section{Footnote}

Reporting Checklist: The authors have completed the MDAR reporting checklist. Available at http://dx.doi.org/10.21037/ apm-20-1811

Data Sharing Statement: Available at http://dx.doi. org/10.21037/apm-20-1811
Conflicts of Interest: All authors have completed the ICMJE uniform disclosure form (available at http://dx.doi. org/10.21037/apm-20-1811). The authors have no conflicts of interest to declare.

Ethical Statement: The authors are accountable for all aspects of the work in ensuring that questions related to the accuracy or integrity of any part of the work are appropriately investigated and resolved. This investigation was a retrospective study and was approved by our institutional review board. The informed consent requirement was waived. All procedures performed in this study involving human participants were in accordance with the Declaration of Helsinki (as revised in 2013).

Open Access Statement: This is an Open Access article distributed in accordance with the Creative Commons Attribution-NonCommercial-NoDerivs 4.0 International License (CC BY-NC-ND 4.0), which permits the noncommercial replication and distribution of the article with the strict proviso that no changes or edits are made and the original work is properly cited (including links to both the formal publication through the relevant DOI and the license). See: https://creativecommons.org/licenses/by-nc-nd/4.0/.

\section{References}

1. Ren HW, Wu Y, Dong JH, et al. Analysis of clinical features and imaging signs of COVID-19 with the assistance of artificial intelligence. Eur Rev Med Pharmacol Sci 2020;24:8210-8.

2. National Health Commission. Novel Coronavirus Pneumonia Diagnosis and Treatment Scheme (trial, 6th edition). (2020-02-19).

3. Holshue ML, DeBolt C, Lindquist S, et al. First Case of 2019 Novel Coronavirus in the United States. N Engl J Med 2020;382:929-36.

4. Kanne JP. Chest CT findings in 2019 novel coronavirus (2019-nCoV) infections from Wuhan, China: Key points for the radiologist. Radiology 2020;295:16-7.

5. Huang C, Wang Y, Li X, et al. Clinical features of patients infected with 2019 novel coronavirus in Wuhan, China. Lancet 2020;395:497-506.

6. Wu Y. Clinical value of hypersensitive creative protein combined with blood routine in diagnosis of bacterial pneumonia, viral pneumonia and mycoplasma pneumonia in children. Chinese Journal of Clinical Laboratory Science 2019;2:83-84. 
7. Das KM, Lee EY, Langer RD, et al. Middle east respiratory syndrome coronavirus: What does a radiologist need to know? AJR Am J Roentgenol 2016;206:1193-201.

8. Choi WJ, Lee KN, Kang EJ, et al. Middle east respiratory syndrome-coronavirus infection: A case report of serial computed tomographic findings in a young male patient. Korean J Radiol 2016;17:166-70.

9. Lv H, Chen T, Pan Y, et al. Pulmonary vascular enlargement on thoracic CT for diagnosis and differential diagnosis of COVID-19: a systematic review and metaanalysis. Ann Transl Med 2020;8:878.

10. Koo HJ, Lim S, Choe J, et al. Radiographic and CT features of viral pneumonia. Radiographics 2018;38:719-39.

11. Xie X, Zhong Z, Zhao W, et al. Chest CT for Typical

Cite this article as: Li XY, Zhou Y, Kong ZX, Hou MD, Luo N, Sun WH, Huang N, Yang C, Zhang AD, Li YS. Exploration of CT manifestations of different clinical types of novel coronavirus pneumonia. Ann Palliat Med 2021;10(1):3744. doi: 10.21037/apm-20-1811
2019-CoV Pneumonia: Relationship to Negative RT-PCR Testing. Radiology 2020;296:E41-E45.

12. Zhou Q, Gao Y, Wang X, et al. Nosocomial infections among patients with COVID-19, SARS and MERS: a rapid review and meta-analysis. Ann Transl Med 2020;8:629.

13. Lv M, Wang M, Yang N, et al. Chest computed tomography for the diagnosis of patients with coronavirus disease 2019 (COVID-19): a rapid review and metaanalysis. Ann Transl Med 2020;8:622.

14. Krafft C, Christy C. Mycoplasma pneumonia in children and adolescents. Pediatr Rev 2020;41:12-9.

(English Language Editor: A. Kassem) 